\title{
Evaluación de la calidad docente y
} promoción del profesorado (V). Legislación universitaria española (c): desarrollo de la Ley Orgánica de Universidades (2002-2005)

Evaluation of teaching and faculty promotion (V). University Spanish Legislation (c): development of the Organic Law of Universities (2002-2005)

\section{Josep Carreras}

Departament de Ciències Fisiològiques I. Facultat de Medicina. Universitat de Barcelona. Barcelona.

La Ley Orgánica de Universidades (LOU) estableció un sistema de selección del profesorado funcionario en dos fases: una fase de habilitación nacional y una fase de concursos de acceso a las plazas convocadas por las universidades. El sistema de habilitación, fue muy criticado por los gobiernos de las Comunidades Autónomas, por la Conferencia de Rectores de las Universidades Españolas (CRUE), por autoridades académicas, profesores, partidos políticos de la oposición y organizaciones sindicales. Se señaló que no respetaba la autonomía universitaria; que no aseguraba que las plazas se cubrieran con las personas más adecuadas; que no eliminaba la "endogamia" en la selección del profesorado, y que resultaba altamente costoso e ineficiente. A la vez, se denunciaron arbitrariedades e irregularidades en el proceder de las comisiones de habilitación. Por todo ello, el Partido Socialista al acceder al gobierno el año 2004 inició un proceso dirigido a la sustitución del sistema de habilitación por un sistema de acreditación.

\footnotetext{
Correspondencia:

J. Carreras

Casanova 143. 08036 Barcelona.

Fax: 934035882

e-mail: jcarreras@ub.edu
}

Por lo que respecta a la selección del profesorado contratado permanente, la LOU determinó que las Universidades debían realizarla entre doctores cuya actividad académica hubiera sido evaluada positivamente por la Agencia Nacional o por una Agencia Autonómica de evaluación. El procedimiento desarrollado por la Agencia Nacional fue duramente criticado; por lo cual el gobierno del Partido Socialista lo modificó, haciéndolo más ágil y transparente. La normativa de los concursos de acceso a plazas concretas quedó fijada por los estatutos de cada universidad; lo que determinó la existencia de notables diferencias entre las mismas.

Palabras clave: Selección del profesorado universitario. Legislación española.

The organic Law of Universities (LOU) established a system with two steps to appoint the members of the bodies of functionary professors: a first step of national "habilitation", and a second step of concourse to the specific positions convoked by the universities. The "habilitation" procedure was censured very much by the Governments of the Autonomous Spanish Communities, the Conference of University Rectors, 
the academic authorities, the professors, the political parties and the labour organizations. It was stated that the procedure did not respect the autonomy of the universities, did not ensure the selection of the best candidates, did not abolish the "endogamy", and that it was very expensive and inefficient. Therefore, when in the year 2004 the Socialist Party formed a new Government, it initiated the substitution of the system of "habilitation" by a system of "accreditation". Regarding to the direct selection of contracted professors by the universities, the LOU established a previous step of evaluation of the academic activity of the candidates by an official agency. As the procedures developed by the National Agency for Evaluation of Quality were censured very much, the Socialist Government changed the system, to improve its efficiency and transparency. The rules of the specific concourses developed by the universities were established by their statute, causing significant differences among them.

Key words: Selection of university professors. Spanish legislation.

\section{INTRODUCCIÓN}

La Ley Orgánica de Universidades (LOU), aprobada por las Cortes Generales en diciembre del año 2001 durante el gobierno del Partido Popular ${ }^{1}$, estableció que el personal docente e investigador de las universidades públicas estaría constituido por funcionarios de los cuerpos docentes universitarios y por personal contratado (Art. 47). Los primeros pertenecerían a los cuatro mismos cuerpos que había determinado la anterior Ley de Reforma Universitaria (LRU) del año 1983: catedráticos de universidad, profesores titulares de universidad, catedráticos de escuela universitaria y profesores titulares de escuela universitaria (Art.56). El personal docente contratado estaría constituido por ayudantes, profesores ayudantes doctores, profesores colaboradores, profesores contratados doctores, profesores asociados, profesores eméritos y profesores visitantes (Art.49-54).

El acceso a los cuerpos docentes universitarios quedó articulado en un doble proceso: una primera fase de habilitación nacional (Art.57-62) y una segunda fase de concursos de acceso internos a las universidades (Art.63). Sólo con posterioridad a la selección por una universidad, al nombramiento y a la toma de posesión se adquiriría la condición de funcionario.

La regulación y el régimen jurídico del personal contratado, cuyo total no podría superar el cuarenta y nueve por ciento del total del personal docente e investigador de la universidad, serían competencias de las Comunidades Autónomas. Su contratación se realizaría mediante concursos públicos (Art.48) y la misma tendría carácter temporal en todos los caso, excepto para los profesores colaboradores y los profesores contratados doctores. Para estas dos categorías de profesorado y para los profesores ayudantes doctores, la contratación exigiría previa evaluación positiva por parte de la Agencia Nacional de Evaluación de la Calidad y Acreditación (ANECA) o del órgano de evaluación externa que la Ley de la Comunidad Autónoma determinase.

En este artículo analizamos primero el desarrollo normativo y los resultados de los sistemas de selección del profesorado funcionario (en sus dos fases, de habilitación y de concursos de acceso), y luego el sistema de selección del profesorado contratado permanente, poniendo especial énfasis en el proceso de evaluación de sus méritos.

\section{SELECCIÓN DEL PROFESORADO FUNCIONARIO}

\section{Fase de habilitación nacional.}

\section{Recursos de inconstitucionalidad}

En la presentación del proyecto de la LOU a las Comisiones del Congreso de los Diputados ${ }^{2}$ y del Senado $^{3}$, a los Plenos de ambas Cámaras ${ }^{4,5}$ y al Pleno del Consejo de Universidades ${ }^{6,7}$, la Ministra de Educación, Cultura y Deporte, Pilar del Castillo, argumentó que el sistema previsto para el acceso a los cuerpos docentes universitarios constituía un sistema más abierto, competitivo, objetivo y transparente que el establecido por la LRU; que al mismo tiempo que garantizaría la calidad del profesorado reforzaría la autonomía universitaria. El sistema supondría un incremento de la autonomía de las universidades, puesto que serían estas las que, con los procedimientos que establecieran para los correspondientes concursos de acceso, seleccionarían a su profesorado de acuerdo con sus políticas y programas de recursos humanos, necesidades e intereses. Al mismo tiempo, constituiría un refuerzo de la calidad, al elegir las universidades entre aquellos que hubieran sido habilitados por un proceso 
transparente y competitivo que garantizaría la libre concurrencia, el rigor y la evaluación del mérito y la capacidad.

No obstante, la opinión del Gobierno en el sentido de que el sistema de selección del profesorado numerario garantizaba la autonomía de las universidades no fue compartida ni por éstas ni por los Gobiernos de las autonomías. Un informe sobre los aspectos de la LOU cuya constitucionalidad era cuestionable, realizado para la Conferencia de Rectores de las Universidades Españolas (CRUE) por un equipo de profesores de la Universidad Carlos III $^{8}$ afirmó: "En determinados casos, especialmente en áreas de conocimiento pequeñas, puede suceder que haya un solo habilitado. En tales casos (con el paso de dos años) dicha persona ha de ser obligatoriamente admitida por la Universidad que convocó la plaza, con lo cual la misma queda sin margen alguno de acción propia en la selección del profesorado. (...). En situación parecida pueden hallarse, aunque haya más de un habilitado por convocatoria, la Universidades que provean en último término. (...) En estos casos se vacía de contenido el derecho a la autonomía universitaria que incluye como elemento fundamental la selección del profesorado. Por lo tanto, en este aspecto concreto la LOU puede considerarse inconstitucional". De forma parecida argumentó un informe elaborado por el "Consell Consultiu de la Generalitat de Catalunya", que señaló que la obligación de proveer las plazas convocadas transcurrido cierto tiempo caso de haber concursantes para las mismas no dejaba el mínimo margen de libertad para las universidades en la selección del profesorado.

Apoyándose en ambos informes, que además del aspecto mencionado referente a la selección del profesorado hallaron en la LOU otros preceptos que pudieran ser inconstitucionales, el PSOE presentó un recurso ante el Tribunal Constitucional, al que se sumaron todos los partidos políticos de la oposición excepto el $\mathrm{PNV}^{10}$. También presentaron recursos de inconstitucionalidad la Junta de Andalucía, el Parlamento Andaluz, la Junta de Comunidades de Castilla-La Mancha, la Asamblea de Extremadura, las Cortes de Aragón, el Parlamento de Navarra y el Gobierno de las Illes Balears ${ }^{11}$. En cambio, el Gobierno de Catalunya decidió no presentar recurso $^{12,13}$, y los votos de Convergencia i Unió y del Partido Popular de Cataluña bloquearon la iniciativa del Parlamento Catalán al respecto ${ }^{14}$.

Por su parte la CRUE se opuso desde el primer momento al procedimiento de selección del profeso- rado universitario establecido por la LOU por "basarse en un sistema de pseudohabilitación que en realidad sería un examen único de ámbito estatal (...) poco respetuoso con la autonomía de las universidades, que pretendía ajustar el número de habilitados al número de plazas convocadas". Presentó como "alternativa a considerar un sistema mixto de acreditación curricular estatal combinada con la especificación de las pruebas de selección del profesorado en los Estatutos de cada Universidad, siempre según los principios de objetividad, capacidad y mérito" ${ }^{15-17}$.

\section{Normativa sobre el sistema de habilitación nacional}

A los tres meses de la publicación de la LOU, en marzo del 2002, el Ministerio remitió el borrador del Real Decreto que debía regular el sistema de habilitación nacional para el acceso a cuerpos de funcionarios docentes universitarios ${ }^{18}$ a la Comisión Académica Ampliada del Consejo de Universidades, para su estudio, análisis y debate. Tres meses más tarde, el 12 de julio, la Ministra presentó al Consejo de Coordinación Universitaria una nueva versión del proyecto del Real Decreto ${ }^{19}$, que recogía parte de las modificaciones que se habían sugerido; entre ellas la de impedir que formasen parte de las comisiones de habilitación los profesores que no estuvieran en activo, posibilidad contemplada en el primer borrador y que había sido duramente criticada por atentar contra la autonomía universitaria ${ }^{20}$. El Consejo de Coordinación Universitaria emitió un informe positivo sobre el proyecto de Real Decreto ${ }^{21}$ que, después de ser dictaminado también positivamente por el Consejo de Estado ${ }^{22}$, fue publicado en el BOE con fecha de 26 de julio ${ }^{23}$.

El Real Decretó precisó que en cada convocatoria el número de habilitados estaría en función del número de plazas convocadas por las universidades, la existencia de vacantes resultantes de concursos de acceso, la existencia de habilitados que no ocupasen plaza y otros criterios relevantes con el fin de garantizar la posibilidad de selección por parte de las universidades (Art.3.2).

Para participar en las pruebas de habilitación para profesores titulares de universidad haría falta estar en posesión del título de doctor y para hacerlo en las pruebas de catedrático de universidad haría falta, además, ser profesor titular de universidad o catedrático de escuela universitaria con tres años de antigüedad; si bien el Consejo de Coordinación Universitaria podría eximir de estos requisitos a 
quienes tuvieran la condición de doctor con, al menos, ocho años de antigüedad (Art. 5.1). Los candidatos que con posterioridad desearan concurrir a los concursos de acceso para ocupar plazas docentes vinculadas a plazas asistenciales de instituciones sanitarias deberían acreditar, también, estar en posesión del título de especialista sanitario procedente y justificar en su currículo los correspondientes méritos asistenciales.

Las comisiones de habilitación estarían constituidas por siete miembros, todos ellos catedráticos de universidad en las pruebas para profesores de este cuerpo; en las pruebas para profesor titular de universidad tres serían catedráticos y cuatro profesores titulares (Art.6.1). Formando parte de las comisiones podría haber un miembro del personal funcionario científico e investigador del CSIC que hubiera sido adscrito a la correspondiente área de conocimiento y un profesor de universidades de otros Estados miembros de la Unión Europea de categoría igual o equivalente a la correspondiente a las plazas objeto de convocatoria. A tal fin, la Comisión Académica del Consejo de Coordinación Universitaria establecería y mantendría actualizado el correspondiente listado (Art.6.6 y 6.7). Para formar parte de las comisiones de habilitación, deberían tener el reconocimiento de al menos dos períodos de actividad investigadora los catedráticos de universidad y los profesores de investigación del CSIC, y de un período los profesores titulares de universidad y los investigadores científicos del CSIC (Art.6.2). No podrían ser miembros de las comisiones los profesores que se hallasen en situación de excedencia o de suspensión de funciones (Art.6.3).

Las pruebas se realizarían en la universidad donde prestase sus servicios o estuviera adscrito el presidente de la correspondiente comisión (Art.10.1). En el acto de presentación los candidatos deberían entregar, en todos los casos, su historial académico, docente e investigador y, en su caso, asistencial-sanitario; así como un ejemplar de las publicaciones y los correspondientes documentos acreditativos. Para las pruebas de profesor titular de universidad deberían presentar, además, un proyecto docente, original y personal, que debería incluir el programa de una o varias asignaturas de una o varias de las materias troncales asignadas al área de conocimiento en las directrices generales de los planes de estudio, y un proyecto investigador (Art.9.1).

En las pruebas para profesor titular de universidad, la primera prueba consistiría en la exposición oral, durante un tiempo máximo de noventa minu- tos, de los méritos e historial, y en la defensa de los proyectos docente e investigador. La segunda prueba consistiría en la exposición oral, durante un tiempo máximo de una hora, de un tema del programa elegido por el candidato de entre tres sacados a suerte, previa preparación durante un tiempo máximo de una hora. La tercera prueba consistiría en la exposición oral, durante un tiempo máximo de noventa minutos, de un trabajo original e inédito de investigación realizado por el candidato, solo o en equipo, en este caso como director de investigació$\mathrm{n}^{\mathrm{a}}$.En las pruebas para catedrático de universidad, las dos pruebas a realizar por el candidato serían equivalentes a las pruebas primera y tercera de las pruebas para profesor titular. En cada prueba, después de la exposición del candidato, la comisión debatiría con el mismo durante un tiempo máximo dos horas, y después de cada miembro de la comisión entregaría al presidente el correspondiente informe razonado, valorando cada candidato. A la vista de los informes emitidos, la comisión realizaría una votación para determinar el paso a la prueba siguiente; para lo cual se requerirían, como mínimo, cuatro votos favorables. Finalizadas todas las pruebas, a la vista de los correspondientes informes emitidos, la comisión procedería a la votación final; siendo propuestos para habilitación los candidatos que obtuvieran más de cuatro votos favorables. La comisión no podría proponer, y por tanto no podrían obtener más de cuatro votos favorables, más candidatos que el número de habilitaciones que hubieran sido objeto de la convocatoria; pero si podría proponer un número inferior, o incluso la no habilitación de candidato alguno (Art.10).

Finalmente, el presidente de la comisión elevaría una propuesta motivada y vinculante al Presidente del Consejo de Coordinación Universitaria, que procedería a la habilitación de los candidatos (Art.11). Contra la resolución de habilitación los candidatos podrían presentar reclamación que sería valorada por una comisión formada por siete catedráticos de universidad, de diversas áreas de conocimiento con, al menos, tres períodos de docencia e investigación reconocidos. Esta comisión examinaría el expediente de las pruebas, valoraría los aspectos puramente procedimentales, y verificaría el efectivo respeto, por parte de la comisión de habilitación, de la igual-

a. La casuística originada por la exigencia de que el trabajo de investigación presentado por el candidato fuera "original" e "inédito" fue comentada en su día por el ex-rector de la Universidad de Barcelona, Carles Solà ${ }^{24}$. 
dad de condiciones de los candidatos y de los principios de mérito y capacidad de los mismos en el procedimiento de habilitación. Finalmente, la comisión de reclamaciones, en el plazo máximo de tres meses, ratificaría o no la propuesta reclamada; en este caso se retrotraería el expediente hasta el momento en que se produjo el vicio, debiendo la comisión de habilitación formular nueva propuesta (Art.12).

\section{Desarrollo de las pruebas de habilitación}

De acuerdo con la normativa descrita, con fecha 6 de noviembre del $2002^{25}$, se publicó la resolución de la Secretaría del Consejo de Coordinación Universitaria por la que se convocaban las primeras pruebas de habilitación. Según la información proporcionada por la Ministra al Pleno de dicho Consejo $^{26}$, las universidades habían solicitado 122 plazas, pero el número finalmente aprobado fue de 210 (166 catedráticos de universidad, 42 profesores titulares de universidad y 2 profesores titulares de escuela universitaria), correspondientes a 72 áreas de conocimiento. Posteriormente, en abril, julio y noviembre del 2003 se publicaron tres nuevas convocatorias $^{27-29}$, y al haberse completado un ciclo anual del sistema de habilitación, la CRUE presentó un informe ${ }^{30}$ en el que se analizaba dicho sistema a fin de "detectar sus deficiencias principales y señalar alguna posibles medidas para corregirlas".

La primera crítica que la CRUE hizo era la de que el sistema de habilitación no había logrado ponerse en marcha con el ritmo deseable. "Resulta evidente la prudencia con que las universidades van comunicando sus nuevas vacantes, en un número claramente inferior al de las vacantes realmente producidas por las jubilaciones, las renuncias o el crecimiento de las universidades españolas", afirmaba el informe. Y señalaba como causa de este hecho el que "las universidades están siendo parcas en comunicar plazas por el riesgo de no poder ejercer de forma efectiva su derecho a escoger, entre un número significativo de profesores habilitados, el más capacitado para el puesto de trabajo a cubrir". Diagnóstico con el que coincidía un miembro de una de las comisiones de habilitación que manifestó públicamente su opinión" ${ }^{31}$ : "Podría darse el caso de que la universidad convocara una plaza pensando en un perfil científico y docente y que ninguno de los habilitados poseyera dicho perfil. (...) Podría suceder que las universidades no convocaran plazas hasta que sus candidatos estuvieran habilitados o intuyeran claramente que lo iban a ser". Por otra parte, la CRUE señaló que el sistema no permitía asegurar que las plazas se cubrían por personas de la especialidad correspondiente a las necesidades de la universidad convocante. La presencia de especialidades muy distintas dentro de una misma área de conocimiento podía imposibilitar, en ciertos casos, la selección del profesor más adecuado para cubrir una vacante y desencadenar una avalancha de recursos. La Conferencia no consideraba razonable que en las áreas de gran amplitud - como es, por ejemplo, la de Medicina - las plazas convocadas debieran cubrirse necesariamente con cualquier candidato habilitado, sin asegurar la existencia de habilitados de todas las especialidades del área.

Además, el informe de la CRUE señaló otras consecuencias de la limitación restrictiva del número máximo de personas a habilitar en cada convocatoria: "Aparte de producir la paralización del sistema (...) está generando frustración entre los aspirantes y falta de confianza entre los miembros de la comunidad universitaria (...); en contra de lo que pudiera parecer a primera vista, alienta las presiones de los habilitados sin plaza, precisamente por tratarse de un número reducido". Opiniones con las que coincidía el profesor antes mencionado ${ }^{31}$, al afirmar por una parte: "No es bueno que se convoquen concursos sabiendo que hay decenas de candidatos y sólo unas pocas plazas para habilitar. El desgaste psíquico y el desembolso económico de los candidatos de seguirse esta senda sería de tal magnitud que pronto no habría candidatos". Y por otra: "Es muy posible que se de el caso de que las personas que obtengan la habilitación antes de optar a las plazas de las universidades que las han generado prefieran convencer a los que mandan en su universidad y esperar, ya habilitados, a que sea ésta la que convoque una plaza".

Pero, probablemente, la crítica más dura que el informe de la CRUE hizo al sistema de habilitación era la de que el mismo resultaba altamente costoso e ineficiente. "A pesar del escaso número de plazas convocadas, el mecanismo previsto por el Real Decreto ocasiona unos costos económicos y humanos totalmente desproporcionados respecto del resultado a obtener", decía el informe, y especificaba: "En diversas áreas, más de un centenar de candidatos compiten por media docena de plazas convocadas en tres convocatorias distintas al año, a razón de dos o tres plazas por convocatoria. Las mismas personas, pues, deben desplazarse a lo largo del país para participar en las mismas pruebas una y otra vez. A su vez, a lo largo del año, 21 profesores son nombrados para formar parte de 3 comisiones ( 7 por comisión) para juzgar a los mismos candidatos". Para 
concluir: "Las consecuencias de este funcionamiento son evidentes: frustración de los candidatos y de quienes los juzgan, sobreesfuerzo de unos y otros, y el colapso de la universidad española, dado que candidatos y juzgadores suelen ser profesores que, durante semanas y hasta meses, se ven imposibilitados para impartir sus clases y realizar su trabajo de investigación habitual”.

Aparte de la CRUE, otros muchos criticaron los altos costes humanos y económicos, y el elevado grado de ineficiencia del sistema de habilitación: miembros de las comisiones ${ }^{31,32}$, rectores y ex-rectores $^{33-35}$, profesores ${ }^{36-39}$ y sindicatos ${ }^{40}$. Especialmente interesante es el análisis realizado por Susana Pérez de Pablos ${ }^{41}$, quien calculó que, dado que en las pruebas de habilitación a realizar el curso 2003-04 participarían 226 comisiones de siete miembros, casi 1600 profesores pasarían un tiempo medio de un mes fuera de su universidad para examinar a los candidatos, y que alrededor de 6,600 aspirantes (unos 2,200 por convocatoria) tendrían que desplazarse por toda España para realizar las pruebas que les permitiesen optar a sólo 576 habilitaciones.

A fin de superar estas deficiencias, la CRUE propuso tres medidas concretas: poner en marcha cuanto antes una convocatoria de habilitación amplia, en todas las áreas de conocimiento, que permitiera que a los concursos de acceso convocados por las universidades pudiera concurrir un número de candidatos habilitados significativo, tendiendo a la no limitación del número de habilitados por convocatoria; agrupar todas las plazas comunicadas a lo largo del año en una sola convocatoria, y celebrar las pruebas de habilitación durante los períodos no lectivos del curso.

En cuanto a las características de las propias pruebas, la CRUE indicó que, para estandarizar algo más la prueba de exposición de un tema, la normativa debía precisar el número mínimo de créditos de las asignaturas cuyos programas debían presentar los candidatos, así como el contenido que debían tener los proyectos docentes. Otros expresaron quejas relativas a la forma "aleatoria" como ciertas comisiones elegían a los candidatos, a las "arbitrariedades" y a las "graves irregularidades" producidas por comisiones cuyos miembros podían tener "menor experiencia y curriculo que los evaluadores" ${ }_{39,42}$. Desarrolladas ya numerosas pruebas de habilitación, Gualberto Buela-Casal realizó un análisis crítico del proceso y de los criterios de evaluación utilizados en las mismas ${ }^{43}$. Este autor destacó la dificultad de la evaluación de varios candidatos, cuando la evaluación previa de la productividad científica de un candidato medio ha requerido, según sus cálculos, 275 evaluadores especializados. Por lo que hace a la primera prueba, consideró como principales problemas la limitación de la fase de debate a 90 minutos (lo que significa que cada miembro de la comisión dispone, como promedio, de 12 minutos de interacción con el candidato), el hecho de que no se hicieran las mismas preguntas a todos los candidatos, la no fijación del valor de la presentación oral y la dificultad de que prospere una reclamación, dado que no existe la obligación de grabar el desarrollo de la prueba. Por otra parte, demostró con ejemplos reales la ambigüedad de los criterios de evaluación que suelen establecer las comisiones de habilitación; la influencia que el número de miembros de la comisión tiene (la legislación permite que las pruebas puedan celebrarse incluso con sólo cuatro miembros) sobre el porcentaje de votos positivos necesarios para superar la prueba $(57 \%$ con una comisión de siete miembros, $100 \%$ con una comisión de cuatro miembros), y las incongruencias que de todo ello derivan, cuestionando la fiabilidad del proceso. Buela-Casal concluyó que era necesario, por una parte, establecer y publicar en el BOE, simultáneamente con la convocatoria de la plaza, los criterios operativos de evaluación, un baremo en el que constara el valor que tiene cada mérito y el procedimiento para la asignación de las puntuaciones $\mathrm{y}$, por otra parte, que las comisiones actuaran siempre con el mismo número de miembros. Consideró, finalmente, que la primera prueba debía mantenerse, si bien su valor debería ser muy limitado. En cambio, consideró innecesaria la segunda prueba de las pruebas de habilitación para catedrático de universidad y la tercera prueba de la habilitación para profesor titular de universidad.

Cabe añadir que, respecto a la casuística que presentan las áreas de gran amplitud, la Federación Nacional de Asociaciones de Catedráticos de Universidad ya había señalado que podía darse el caso de que entre los siete miembros de una comisión no hubiera especialistas de la materia sobre la que algún concursante hubiera presentado sus trabajos y su programa ${ }^{44}$.

Tanto las autoridades ministeriales ${ }^{2-7}$ como aquellos que habían defendido el sistema de habilitación $^{45-49}$ habían señalado como uno de los objetivos fundamentales de dicho sistema conseguir la erradicación de la "endogamia" a que había conducido la LRU en la selección del profesorado. Sin embargo, desde un primer momento, hubo quienes opinaron que lo que haría la LOU sería sustituir un tipo de endogamia por otro. Así, durante la fase de tramita- 
ción parlamentaria de la Ley, el representante del Bloque Nacionalista Galego había manifestado²: "Las pruebas de habilitación (...) no van a hacer que desaparezca ninguno de los efectos de la coaptación endogámica, sino que la van a plantear en un terreno más reducido del profesorado". El representante del Grupo Parlamentario de Coalición Canaria había denunciado que el sistema de habilitación podía "degenerar en una cultura de escuelas dominantes o de universidades dominantes" ${ }^{2} \mathrm{y}$, en el mismo sentido, el representante del Grupo Parlamentario Socialista había señalado que "los potenciales miembros de las comisiones de habilitación estarán, concentrados en su mayoría en muy pocas universidades, justamente en aquellas que tienen mayor número de doctores ${ }^{50 "}$.Por otra parte, Francisco Michavila, director de la Cátedra UNESCO de Gestión y Política Universitaria había escrito ${ }^{51}$ : "El control local que se denuncia ahora se substituiría por otro, acaso más perverso, a cargo de quienes controlan las áreas de conocimiento". Alfredo Pores, catedrático de Física de la Universidad Autónoma de Madrid había destaca$\mathrm{do}^{52}$ : "Este análisis peca incluso de optimista, ya que olvida las posibilidades de comportamientos coherentes -las confabulaciones-. Ejemplo de ellas son las famosas y de todos conocidas escuelas que florecieron al amparo de los sistemas de selección basados en loterías durante el franquismo". Y, en el mismo sentido, había manifestado José Contreras, catedrático de Antropología de la Universidad de Barcelona ${ }^{53}$ : "Tal como está planteado el anteproyecto de ley, no va a cambiar nada en absoluto. (...) Suponiendo que la actual endogamia (si es que solo hubiera un tipo, que no es el caso) fuera de aldea, la endogamia que se planteará con el sistema de habilitación será de clan.(...) Según que el sorteo favorezca a uno u otro clan, ya se podrá plantear que candidatos son los que tienen más posibilidades de ser habilitados. En definitiva, vamos a sustituir una selección del profesorado que está en manos de las mafias departamentales por otra que estará en manos de las mafias estatales". Si bien el resultado de las pruebas de habilitación ya ha suscitado críticas en este sentido, solo el análisis comparativo de la composición de las comisiones con los listados de habilitados podría permitir un juicio objetivo. De todas formas, es significativo que ya en el informe de la CRUE ${ }^{30}$ se recomendara: "Para mejorar la objetividad del proceso y prevenir la endogamia de área, se ha de garantizar la rotación de vocales en las comisiones que resuelven las pruebas de habilita- ción, evitando que los mismos profesores formen parte de ellas una vez tras otra".

Entre los partidos políticos de la oposición, el Partido Socialista e Izquierda Unida manifestaron sintonía con los planteamientos de la CRUE, lo mismo que los sindicatos CCOO y UGT ${ }^{54}$. En cambio, el Secretario de Estado de Educación y Universidades consideró desacertadas gran parte de las denuncias contenidas en el informe de la CRUE ${ }^{54,55}$. Ello no obstante, el Ministerio y la CRUE acordaron evaluar, mediante la creación de una comisión de trabajo conjunta, los resultados de las habilitaciones para introducir posibles mejoras ${ }^{56,57}$. Como resultado de los acuerdos preliminares alcanzados en dicha comisión, el Ministerio se comprometió a realizar una sola convocatoria anual de las pruebas se habilitación entre los meses de junio y septiembre, y a que ningún vocal pudiera ser miembro de dos comisiones consecutivas ${ }^{58}$.

Digamos para acabar este apartado que, frente a tan diversas críticas, la única ventaja del sistema de habilitación que hemos encontrado explicitada en la prensa es la derivada del hecho de que los tribunales tendrían ante sí, por primera vez desde hace mucho, a todos los posibles candidatos existentes en todo el ámbito estatal, con lo que ello significa de variedad y riqueza intelectual ${ }^{31}$. Las autoridades ministeriales habían destacado que el sistema de habilitación facilitaría la movilidad del profesorado entre las universidades, al recuperarse la posibilidad de traslado mediante la participación en los concursos de acceso sin tener que realizar nuevas pruebas ${ }^{2,3,6,7}$. Pero esta supuesta ventaja llegó a ser incluso considerada como un posible peligro para la imparcialidad de las comisiones de habilitación, cuyos miembros podrían ver futuros competidores en los habilitados. Y así se llegó a sugerir que debería modificarse la normativa para que los miembros de una comisión no pudieran concurrir a una de las plazas que hubieran dado origen a la convocatoria; para evitar que fueran los primeros interesados en no habilitar a los mejores candidatos, que podrían ser más tarde posibles competidores ${ }^{39}$.

\section{Cambio de partido en el Gobierno. Modificación del sistema de habilitación}

La proximidad de las elecciones generales, a celebrar en marzo del 2004, determinó que los diversos partidos políticos explicitaran sus intenciones respecto a la LOU. El Partido Popular consideraba la Ley como un asunto cerrado y sólo se proponía modificar aspectos mínimos, como los arriba comen- 
tados. El candidato a la presidencia de gobierno del PSOE afirmó reiteradamente la intención de los socialistas de reformar la LOU si ganaban las elecciones, suprimiendo, entre otros, los artículos que vulneraban la autonomía de las universidades y sustituyendo el sistema de habilitación por una acreditación otorgada según el curriculo. Izquierda Unida propuso la inmediata introducción de modificaciones transitorias y la derogación total de la LOU a medio plazo, sustituyendo la habilitación actual por una curricular sin límite de número ${ }^{59,60}$.

Tras la victoria del PSOE, el nuevo Presidente de Gobierno, José Luis Rodríguez Zapatero, en el discurso de investidura reiteró su compromiso de reformar la LOU, lo que determinó que la CRUE ofreciera al Gobierno su apoyo y colaboración ${ }^{61}$. Se inició entonces un proceso que culminaría, en septiembre del año 2006, con la remisión al Congreso de los Diputados del proyecto de ley por el que de modificaba la $\mathrm{LOU}^{62}$.

Pero, mientras tanto, se hizo evidente la necesidad de reformar algunos aspectos de la normativa de las pruebas de habilitación, a la espera de la nueva ley. Así lo solicitó la CRUE ya en el mes de junio del año $2004{ }^{63}$, indicando la conveniencia de "mantener las pruebas, agrupando convocatorias y ampliando el número de plazas, de modo que se garantizase la función habilitadora y mejorase la eficiencia del sistema". Pidió, además, la CRUE que, de acuerdo con su informe aprobado meses antes ${ }^{30}$, se garantizase la rotación de los miembros de los tribunales y la adecuada definición del contenido de las pruebas. También, el mismo mes, la FE.CC.OO propuso diversas modificaciones de la normativa de habilitación: eliminar la limitación del número de habilitados; incluir a un representante sindical del profesorado en los tribunales de habilitación; establecer pruebas específicas de habilitación para promoción interna y concursos de promoción interna entre habilitados de la propia universidad ${ }^{64}$. Y, unos meses más tarde, la "Asociación para el avance de la ciencia y tecnología en España" (AACTE) propuso como mejora del sistema de habilitación que las universidades especificaran a qué especialidad correspondía cada plaza solicitada y las comisiones habilitasen sólo a candidatos de dichas especialidades; que como miembros de dichas comisiones sólo pudiesen formar parte los profesores e investigadores con mayor número de sexenios (primer quartil del área); que los mismos se comprometiesen de forma explícita y legalmente vinculante a abstenerse de evaluar a candidatos con los que existieran intereses personales; que las pruebas de habilitación incluyeran el desarrollo de una clase sobre un terma del área elegido entre seis seleccionados al azar, y que los concursos de acceso de las universidades se limitaran a conceder la plaza al habilitado en la especialidad mejor evaluado en la habilitación ${ }^{65}$.

En su primera reunión con el Pleno del Consejo de Coordinación Universitaria, celebrado en el mes de julio, en la que expuso las líneas de actuación del Gobierno para sustituir el sistema de habilitación, la Ministra de Educación y Ciencia, $\mathrm{M}^{\mathrm{a}}$ Jesús San Segundo, aceptó que, a la espera de haber culminado la reforma, se haría imprescindible arbitrar medidas urgentes para la provisión de plazas, modificando la normativa vigente ${ }^{6}$. A tal fin, el Gobierno elaboró un Real Decreto cuyo borrador fue presentado al Consejo de Coordinación Universitaria ya en octubre del año $2004^{67,68}$ si bien no se publicó hasta abril del año siguiente ${ }^{69}$. Dicho decreto estableció una única convocatoria anual en vez de las tres existentes hasta entonces; simplificó los trámites y suprimió el carácter de "inédito" de los trabajos de investigación presentados por los candidatos. Y en aplicación del mismo, el mes de septiembre del año 2005 se publicó la octava convocatoria de pruebas de habilitación ${ }^{70,71}$, que incluía 2,162 habilitaciones (606 de catedrático de universidad y 1,502 de profesor titular de universidad) para atender a las 482 plazas (228 de catedrático y 229 de profesor titular) solicitadas por las universidades. En las siete convocatorias anteriores (25-29,72-74) se había habilitado a un total de 509 candidatos; entre ellos, 186 profesores titulares de universidad y 307 catedráticos de universidad, a través de 735 y 639 pruebas, respectivamente (75). Estas siete convocatorias incluyeron 70 plazas de profesor titular y 101 plazas de catedrático de áreas de conocimiento relacionadas con las facultades de medicina.

Comentemos, para finalizar este apartado, que por lo que respecta al aspecto económico, las pruebas de habilitación habían resultado muy costosas. El propio Ministerio del Gobierno Socialista calculó que organizar en una universidad unas pruebas de habilitación costaba entre 65,000 y 80,000 euros ${ }^{76}$. Y la Secretaría General del Consejo de Coordinación Universitaria, a partir de una estimación directa de los costos económicos realizada en base a las seis primeras convocatorias de habilitación, concluyó que el promedio para cada comisión era algo superior a los 18,000 euros; lo que significaba que cada plaza había costado, aproximadamente, un millón de pesetas ${ }^{77}$. A estos costos directos habría que aña- 
dir los costos indirectos derivados de la necesidad de sustituir repetidamente a los profesores que participaban en las pruebas. Pérez de Pablos ${ }^{78}$ concluyó que si se consideraba lo que costaba a una universidad prescindir de un profesor para que pudiera participar en las pruebas, junto con los gastos de desplazamiento, alojamiento y manutención, se llegaba a una media de unos mil euros por habilitación. De todas formas, puede ser cierto, como comentó la AACTE ${ }^{65}$, que el coste económico del sistema de acceso a los cuerpos docentes establecido por la LRU hubiera sido muy superior al del sistema de habilitación, si en vez de presentarse a las pruebas sólo candidatos "de casa" hubieran concursado todos los que han optado a la habilitación.

\section{Fase de concursos de acceso}

En desarrollo de la LOU, el Real Decreto que reguló el sistema de habilitación ${ }^{23}$ que la convocatoria de los concursos de acceso determinó a cuerpos docentes especificaría las plazas objeto de concurso, señalando la categoría del cuerpo, el área de conocimiento y, en su caso, las actividades docentes e investigadoras referidas a una materia de un título oficial que debería realizar quien obtuviera la plaza. En ningún caso se podría hacer referencia a orientaciones sobre la formación de los posibles candidatos o cualesquiera otras que vulnerasen los principios de igualdad, mérito y capacidad para el acceso a la función pública o establecieran limitaciones a los derechos de los funcionarios reconocidas por las leyes (Art.14.3). En el caso de plazas docentes vinculadas a plazas asistenciales de instituciones sanitarias, únicamente podrían participar en los concursos de acceso, cuando la plaza vinculada fuera de especialista, los habilitados que ostentaran el correspondiente título oficial de la especialidad (Art.15.1).

Los concursos de acceso serían resueltos, en cada universidad, por una comisión constituida al efecto, de acuerdo con el procedimiento previsto en sus estatutos. Dicho procedimiento se basaría en criterios objetivos y generales, y garantizaría, en todo caso, la plena competencia docente e investigadora de los miembros de la comisión, así como que la categoría funcionarial de los mismos fuera igual, equivalente $o$ superior a la plaza objeto de concurso. Por otra parte, los miembros de las comisiones deberían contar con el reconocimiento de los mismos períodos de investigación mínimos exigidos para los miembros de las comisiones de habilitación (Art.16.1). En los concursos para plazas docentes vinculadas a plazas asistenciales de instituciones sanitarias, dos de los miem- bros de la comisión, que deberían ser doctores (salvo en el caso de plazas de profesores titulares de escuelas universitarias) y deberían estar en posesión del título de especialista exigido para concursar a la plaza, serían elegidos por sorteo público por la institución sanitaria correspondiente, entre el censo público que anualmente debería comunicar al Consejo de Coordinación Universitaria (Art.16.2).

La universidad debería hacer pública la composición de las comisiones, así como los criterios para la adjudicación de las plazas, que no podrían incluir criterio alguno que impidiera la participación en el concurso de un habilitado (Art.16.1). En el plazo máximo de dos años las plazas convocadas deberían proveerse siempre que hubiera algún concursante a las mismas (Art.17.1). Contra la propuesta de la comisión, los candidatos podrían presentar reclamación ante el rector (Art.18.1), que sería valorada por una comisión de reclamaciones formada por siete catedráticos de universidad, de diversas áreas de conocimiento, con amplia experiencia docente e investigadora, designados en la forma que establezcan los estatutos (Art.18.2). La plaza obtenida tras un concurso de acceso debería desempeñarse al menos durante dos años antes de poder participar en un nuevo concurso a efectos de obtener una plaza de igual categoría y de la misma área de conocimiento en la misma o en otra universidad (Art, 17,7).

Los estatutos de las universidades, al determinar el procedimiento por el que han de regirse los concursos de acceso a cuerpos de funcionarios docentes, y las normativas específicas que al respecto se han establecido difieren por lo que hace a aspectos relativamente importantes, como son el número de miembros de las comisiones, su procedencia y la forma de designación. Así, en el caso de las siete universidades públicas de Cataluña $a^{79-93}$ las comisiones están constituidas por cinco miembros en todos los casos, excepto en dos en que los miembros se reducen a tres. La presencia de profesores de otras universidades españolas o incluso de otros países de la Unión Europea es permitida por algunas universidades y obligada por otras. En tres casos se permite que el personal investigador funcionario del CSIC forme parte de las comisiones. Una universidad establece que las comisiones podrán tener asesoramiento externo y otra determina la incorporación, con voz pero sin voto, de un miembro académico de la universidad designado por la representación sindical.

Por lo que respecta a la elección de los miembros de las comisiones, se establece, en mayor o menor grado, la participación de los departamentos en 
todos los casos; de los centros implicados, en cinco casos; de comisiones delegadas del Consejo de Gobierno de la Universidad, en otros cinco, y del rector en tres. La situación más extrema la constituyen las Universidades de Girona y de Lleida que establecen que los cinco miembros son propuestos por el departamento. Atendiendo a la normativa vigente a nivel estatal, los estatutos de las universidades modifican la composición de las comisiones de acceso a plazas docentes vinculadas a plazas asistenciales de instituciones sanitarias; las cuales proponen o bien dos de los miembros que normalmente son propuestos por otras instancias, o bien dos miembros adicionales.

Finalmente, por lo que hace al procedimiento de los concursos, que debe ser también regulado por los estatutos de las universidades, en todos los casos se establece, como primera fase, la presentación por los concursantes de su historial, de una memoria con su proyecto académico y de los documentos acreditativos correspondientes. Después de la valoración de los mismos por la comisión, como segunda fase, se establece una única prueba de carácter público, consistente o bien en una entrevista del candidato con la comisión (Universidad de Lleida) o bien en una exposición oral por parte del concursante seguida de debate con la comisión. La Universidad Pompeu Fabra exige que los candidatos proporcionen en el acto de presentación los nombres de cinco investigadores de reconocido prestigio internacional a los que la comisión pueda, si se da el caso, pedir referencias, y establece que sólo si así se ha anunciado en la convocatoria se realizará una prueba consistente o bien en una entrevista o en un seminario dado por el candidato.

Señalemos, para acabar, que todas las universidades catalanas determinan que al comenzar el concurso las comisiones deben hacer públicos los criterios y los correspondientes baremos de evaluación , en base a los cuales se hará la propuesta razonada de provisión de plaza. Las normas publicadas con carácter general suelen indicar que deberá valorarse la competencia disciplinaria específica, la capacidad docente e investigadora (así como la competencia asistencial en las plazas vinculadas a instituciones sanitarias) y la adecuación a las prioridades docentes y de investigación de la Universidad, y al perfil de la plaza. En el caso de la Universidad Autónoma de Barcelona, se indica que también se considerará la experiencia en gestión universitaria $y$, en el marco de la normativa vigente, el suficiente conocimiento de las dos lenguas oficiales en
Cataluña a fin de preservar el derecho de los estudiantes a utilizarlas indistintamente.

\section{SELECCIÓN DEL PROFESORADO CONTRATADO PERMANENTE}

\section{Fase de acreditación}

Como hemos indicado previamente, dos son las categorías de profesorado contratado permanente previstas por la LOU: profesores contratados doctores y profesores colaboradores. Pero, dado que sólo los primeros forman parte del profesorado de las Facultades de Medicina, sólo vamos a referirnos a los mismos.

Según el artículo 52 de la LOU, la contratación por las universidades públicas bajo la figura de profesor contratado doctor exige estar en posesión del título de doctor, haber desarrollado al menos tres años de actividad docente e investigadora o prioritariamente investigadora postdoctoral y la previa evaluación positiva por la Agencia Nacional de Evaluación de la Calidad y Acreditación (ANECA), creada de acuerdo con los artículos 31 y 32 de la propia LOU, o por los órganos de evaluación que la Ley de las Comunidades Autónomas determine, en el ámbito de sus respectivas competencias

\section{Ámbito estatal}

En desarrollo del artículo 52 de la LOU, el Real Decreto 1052/2002 de 11 de octubre ${ }^{94}$ reguló el procedimiento para la obtención de la evaluación de la ANECA y de su certificación. Dicho decreto estableció que la evaluación positiva tendría efecto para todas las universidades españolas y no estaría sujeta a plazo de caducidad alguno; de ser negativa, no podría solicitarse una nueva evaluación hasta transcurrido un plazo de seis meses. Estableció, también, una serie de determinantes relativos al procedimiento de avaluación; entre otros, que las evaluaciones fueran realizadas por evaluadores independientes y expertos en el campo científico que correspondiera, y que los criterios de evaluación fueran elaborados con carácter general por la ANECA . La correspondiente resolución, de fecha 30 de octubre de $2002^{95}$, especificó que los grupos de méritos evaluados serían cinco: experiencia investigadora, experiencia docente, formación académica, experiencia y otros méritos profesionales, y otros méritos. Además, detalló los méritos que serían considerados dentro de cada apartado; pero no precisó cuantificación alguna para ellos, lo cual sería una de las causas de falta de transparencia de que se acusó al sistema de evaluación. 
Abierto el plazo de presentación de solicitudes en noviembre del mismo año $0^{96}$, la ANECA creó cinco comités de evaluación para campos científico-técnicos específicos (Ciencias Sociales y Jurídicas, Ciencias Experimentales, Ciencias de la Salud, Enseñanzas Técnicas y Humanidades), constituidos por siete o nueve miembros más el presidente que actuaría con voz pero sin voto, y estableció las normas de procedimiento interno $0^{97}$. Tal como se explica en la Memoria referente al primer año de actividad del "Programa de Evaluación del Profesorado" publicada por la propia $\mathrm{ANECA}^{98}$, el principal propósito de dicho programa durante sus primeros meses de actividad fue el de lograr, para cada una de las figuras de profesorado contratado establecidas por la LOU, una interpretación suficientemente armónica de los criterios de evaluación de méritos en todos y cada uno de los comités de evaluación; así como el establecimiento de unas reglas y estándares de calidad también armonizados. Todo ello teniendo en cuenta las naturales diferencias y peculiaridades existentes en cada uno de los grandes acampos científico-técnicos y en sus respectivas comunidades.

Las reglas y estándares de calidad utilizados durante el primer año de funcionamiento del programa de evaluación ${ }^{98}$, adecuadamente sistematizados, serían luego incorporados a las "Normas de Procedimiento" de la ANECA ${ }^{99}$. Dichas normas determinaron que en el caso de los aspirantes a plazas de Profesor Contratado Doctor, a los que la LOU exigía haber desarrollado al menos tres años de actividad docente o investigadora, o prioritariamente investigadora postdoctoral, era esta actividad la que había de ser específicamente evaluada. A tal fin, se considerarían preferentemente, y por este orden, los méritos de experiencia investigadora y de experiencia docente de carácter postdoctoral. Se valorarían, también, los méritos de formación académica, los méritos de experiencia profesional, y otros méritos, con este orden de prelación, y teniendo en cuenta la posibilidad de valorar dentro de estos criterios la actividad docente e investigadora predoctoral.

El criterio "Experiencia investigadora" referido al periodo posdoctoral se consideraba el elemento prioritario y por tanto de mayor peso en la evaluación para esta figura de profesorado. En principio, se valoraría positivamente, en aplicación de este criterio, una actividad investigadora postdoctoral intensa, desarrollada de forma regular y coherente. Serían méritos a valorar: las publicaciones científicas internacionales con proceso anónimo de revisión por pares y otras publicaciones científicas de reco- nocido valor; las patentes, modelos de utilidad y otros resultados de investigación; la participación en proyectos de investigación obtenidos en convocatorias públicas y competitivas, y la participación en contratos de investigación; las estancias de investigación en el extranjero; las contribuciones a congresos y conferencias científicas, y la dirección de tesis doctorales. En el campo de las Ciencias de la Salud, como en los campos de Ciencias Experimentales y de Enseñanzas Técnicas, las publicaciones científicas se valorarían prioritariamente atendiendo al valor del índice de impacto y al lugar que ocupara la revista en el conjunto de las correspondientes a un mismo campo de conocimiento. Para la valoración positiva en este criterio, se consideraría como estándar, la aportación de entre 5 y 9 publicaciones.

El criterio "Experiencia docente" referido al periodo postdoctoral se consideraba el segundo criterio de mayor peso para la evaluación. En principio, se valoraría positivamente el desarrollo de una actividad docente universitaria extensa, equivalente a un curso académico, y suficientemente activa en materias regladas de grado y, excepcionalmente, de postgrado. Serían méritos a valorar: la amplitud e intensidad de la docencia en el campo disciplinar propio; los ciclos y las instituciones en las que se hubiera ejercido, y las evaluaciones sobre su calidad; la formación didáctica para la actividad docente, la utilización de nuevas tecnologías y la participación en proyectos de innovación docente, y el material elaborado de carácter relevante. En el campo de las Ciencias de la Salud sería mérito relevante, en las áreas clínicas, la posesión del título de especialista.

El criterio "Formación académica" valoraría como méritos: los resultados de la formación académica; la calidad de las instituciones y los programas; las becas, bolsas y ayudas de carácter competitivo recibidas, y la actividad académica, docente o investigadora predoctoral. El criterio "Experiencia y otros méritos profesionales" valoraría el interés y la relevancia práctica que para la docencia y la investigación tuviera la experiencia profesional del solicitante. Y el criterio "Otros méritos" valoraría cualquier otro mérito docente, investigador, profesional o de gestión universitaria no valorado en aplicación de los otros criterios.

Los solicitantes con una excelente experiencia investigadora podrían ser objeto de valoración global positiva aun cuando su experiencia docente no fuera de la misma entidad; aunque la actividad investigadora no podría compensar la ausencia de 
actividad docente, ni viceversa. Los méritos evaluados en los otros tres criterios podrían compensar insuficiencias no relevantes en relación con la experiencia investigadora y la experiencia docente; pero, en ningún caso, suplirían carencias importantes de las mismas.

Las críticas recibidas por el proceso evaluador desarrollado por la ANECA fueron muy numerosas y de origen muy diverso. Profesores, organizaciones sindicales, partidos políticos e, incluso, el presidente de la CRUE y el Defensor del Pueblo criticaron muy duramente la labor de la Agencia; por sus supuestas arbitrariedades, opacidad, utilización de criterios poco adecuados y por la situación de indefensión en que quedaban los docentes ${ }^{100-114}$. En noviembre del año 2003 tuvieron lugar concentraciones de protesta en varias universidades que reunieron, según sus convocantes, a más de 1,500 profesores ${ }^{109,110}$. Pocos días después se publicó un manifiesto que obtuvo centenares de adhesiones ${ }^{111,112}$, y el Partido Socialista e Izquierda Unida llegaron a pedir el cese del director de la ANECA $^{105,106}$.

Por todo ello y habiendo ganado las elecciones generales el Partido Socialista, con el fin de mejorar el procedimiento a seguir por la ANECA, a lo largo del último cuatrimestre del año 2004 se solicitó la colaboración de un grupo de expertos externos. El trabajo desarrollado consistió en la presentación de un diseño de criterios comunes e intervalos para los mismos, especificando los principios y reglas para su aplicación y sus ponderaciones concretas para cada figura contractual. Por otra parte, se recopiló información sobre los criterios de evaluación del profesorado utilizados por las agencias de diversas Comunidades Autónomas. Como resultado de este proceso, mediante Resolución de la Dirección general de Universidades, publicada el mes de marzo del año $2005{ }^{115}$, se modificaron determinados aspectos del sistema de evaluación con el fin de hacerlo más ágil, sencillo y transparente.

Desde diciembre del año 2002 a julio del año 2005 la ANECA evaluó 20,552 solicitudes; de las cuales $5,637(27 \%)$ correspondieron a la figura de profesor contratado doctor. De éstas, un $61 \%$ merecieron evaluación positiva; porcentaje que se elevó a un $63 \%$ al reconsiderarse las resoluciones recurridas (43\%). En el ámbito de las Ciencias de la Salud, se evaluó un total de 2,414 solicitudes, 803 de las cuales correspondieron a la figura de profesor contratado doctor. Recibieron inicialmente evaluación positiva un $45 \%$; porcentaje que ascendió al $52 \%$ por reconsideración de los recursos $(46 \%)^{116}$.

\section{Ámbito autonómico}

Atendiendo a las previsiones de la LOU, diversas Comunidades Autónomas con competencias sobre la enseñanza universitaria desarrollaron sistemas propios para la evaluación del profesorado con vistas a su contratación por las universidades ${ }^{117}$. Describiremos brevemente sólo el caso de Cataluña por las peculiaridades que presenta como consecuencia de haber establecido, mediante la "Llei d'Universitats de Catalunya" ${ }^{118}$, dos categorías distintas de profesor contratado permanente, dentro de la figura de profesorado contratado doctor definido por la LOU: el profesor agregado y el catedrático contratado.

El profesor agregado es la primera categoría superior en la cual se dispone de un contrato indefinido. Para aspirar a la misma se requiere, además de poseer el título de doctor, acreditar un mínimo de tres años de actividad docente y de investigación, o prioritariamente de investigación postdoctoral; así como dos años de actividad docente o investigadora, predoctoral o postdoctoral, o de transferencia de tecnología o de conocimientos, en situación de desvinculación académica de la universidad convocante. Se requiere, también, disponer de la acreditación de investigación emitida por la "Agencia per a la Qualitat del Sistema Universitari de Catalunya" (AQU Catalunya), reconociendo una probada capacidad investigadora $\mathrm{y}$, si así lo ha precisado la universidad en la convocatoria de la plaza, de un informe sobre la actividad docente ${ }^{119}$.

El catedrático es la categoría contractual con carácter indefinido que corresponde a la etapa más avanzada de la carrera académica. Para aspirar a la misma se requieren los mismos requisitos que en el caso de profesor agregado con la diferencia de que la acreditación emitida por AQU Catalunya debe ser la "acreditación de investigación avanzada", que reconoce una carrera de investigación consolidada ${ }^{119}$.

Para la concesión de las acreditaciones mencionadas, AQU Catalunya creó una Comisión de Evaluación de la Investigación y seis Comisiones específicas para los diversos ámbitos: Humanidades, Ciencias Sociales, Ciencias, Ciencias de la Vida, Ciencias Médicas y de la Salud, Ingeniería y Arquitectura. En las primeras convocatorias, en todos los casos, se evaluaron cinco apartados de méritos: publicaciones, dirección y participación en proyectos de investigación, actividad formativa, transferencia de tecnología, patentes y modelos de actividad, y otros méritos. En el caso de las Ciencias Médicas y de la Salud, la ponderación relativa de los cuatro primeros apartados fue, respectivamente, de un $65 \%, 15 \%, 10 \%$ y $10 \%$. Las 
diferencias entre la "acreditación de investigación" y la "acreditación de investigación avanzada" radicarían en los estándares mínimos exigidos en uno y otro caso. Así, digamos a título de ejemplo por lo que hace a las publicaciones en revistas, que en el caso de la acreditación de investigación se exigirían, en principio, un mínimo de 20 publicaciones en revistas indexadas, la mitad de las cuales deberían hallarse en el primer quartil del área y de la mitad de las cuales el candidato debería ser o bien el primer autor o el autor responsable. Para la acreditación de investigación avanzada se requeriría, en principio, 40 publicaciones, de las cuales alrededor de la mitad deberían pertenecer al primer quartil del área ${ }^{120}$.

$\mathrm{Si}$ se comparan los criterios utilizados por la ANECA y por AQU Catalunya, se advierte que una diferencia fundamental es el peso concedido a la evaluación de la actividad docente; significativo para la primera agencia y nulo para la segunda. Ello es así por qué la agencia catalana decidió dejar la evaluación de las actividades docentes y de gestión en manos de las comisiones "ad hoc" de las universidades. Pero, como ya se ha indicado, si la universidad así lo establece en la convocatoria de los concursos de acceso a plazas de profesor agregado o de catedrático, puede requerirse que los candidatos presenten una certificación o evaluación de su actividad docente. Esta certificación será emitida por la Comisión específica para la valoración de los méritos y actividades individuales de AQU Catalunya, que considerará, como criterio preferente, la valoración del autoinforme del candidato, del encargo de actividad docente del quinquenio, del desarrollo y de la actuación profesional, y de la satisfacción de estudiantes y graduados $^{121}$.

Añadamos, para finalizar este apartado, que en las tres convocatorias correspondientes a los años 2003 y 2004 AQU Catalunya realizó 1,103 evaluaciones para "acreditación de investigación" y 359 evaluaciones para "acreditación de investigación avanzada"; de las cuales fueron positivas 451 y 193 , respectivamente. Del total de solicitudes evaluadas de los dos grupos, 317 y 127, respectivamente, correspondieron al ámbito "Ciencias Médicas y de la Salud"; siendo positivas 157 y 57, respectivamente $^{122}$. Por lo que hace a la certificación de la actividad docente, cabe indicar que el proceso se inició el año 2004 , con 8 solicitudes informadas positivamente ${ }^{123}$.

\section{Fase de concurso}

Al comparar las normas establecidas por las distintas universidades, por lo que hace al número de miembros de las comisiones, su procedencia y forma de designación, se advierte que entre las mismas hay el mismo tipo de diferencias que se han señalado antes en el caso de las comisiones de acceso para cubrir las plazas de los cuerpos docentes universitarios. En el caso de las siete universidades públicas de Cataluña ${ }^{124-130}$, los dos tipos de comisiones se constituyen siguiendo normativas análogas, con la diferencia de que en el caso de las comisiones para plazas de profesorado contratado pueden formar parte de las mismas profesores contratados junto a profesores funcionarios.

Por lo que respecta al procedimiento de desarrollo de los concursos, todas las universidades catalanas exigen que, junto con su historial y los documentos acreditativos correspondientes, los candidatos presenten un proyecto académico que debe incluir un plan de actuación docente y, en la mayoría de los casos, un plan de investigación. En el caso de la Universidad de Barcelona se especifica que, para las plazas de catedrático, el plan de investigación debe incluir propuestas de configuración de equipos con las respectivas propuestas de trabajo. Y la Universidad Autónoma de Barcelona requiere la presentación de una memoria justificativa de la adecuación del candidato al perfil de la plaza convocada.

Tres de las universidades catalanas estructuran el concurso en base a tres pruebas, todas ellas públicas y de carácter eliminatorio. La Universidad Autónoma de Barcelona ${ }^{124}$ establece que la primera prueba consiste en la exposición oral de los méritos y de la adecuación a los criterios establecidos en la convocatoria. La segunda prueba consiste en la exposición de una unidad docente del programa elegida por el candidato entre tres propuestas por la comisión; especificando los conceptos teóricos que se quieren transmitir a los alumnos, y la metodología docente y de evaluación que se utilizarán. La tercera prueba consiste en la exposición de un trabajo de investigación.

La Universidad de Barcelona ${ }^{125}$ y la Universidad Politécnica de Cataluña ${ }^{127}$ establecen que la primera prueba consiste en la exposición oral de los méritos, historial y plan de actuación docente; la segunda en la exposición de un tema del programa o programas incluidos en el plan docente, elegido por el propio candidato libremente (Universidad de Barcelona) o entre tres temas seleccionados por sorteo (Universidad Politécnica de Cataluña), y la tercera prueba en la exposición del plan de investigación.

Otras tres universidades catalanas - Universidad Pompeu Fabra ${ }^{128,129}$, Universidad Rovira Virgili ${ }^{130}$ y Universidad de Lleida ${ }^{126}$ - estructuran el concurso 
en sólo dos pruebas, también de carácter eliminatorio en los dos primeros casos. La primera prueba consiste en la exposición oral de los méritos, historial y proyecto académico. La segunda prueba, en el caso de la Universidad Pompeu Fabra y de la Universidad Rovira Virgili, consiste en la exposición de un trabajo de investigación. En el caso de la Universidad de Lleida consiste también en la exposición de un trabajo de investigación en las plazas de catedrático, o de un tema docente en las plazas de profesor agregado.

En todos los casos, se determina que al constituirse la comisión se hagan públicos los criterios específicos y los baremos que se utilizarán para la valoración de los méritos, así como las puntuaciones o votos favorables mínimos requeridos para superar cada prueba y el concurso, en caso de que la universidad no lo haya establecido con carácter general.

En el momento de escribir este artículo no disponemos de datos suficientes para analizar críticamente el desarrollo del proceso de selección del profesorado contratado permanente; ni siquiera en el ámbito de las universidades catalanas, dado que las convocatorias realizadas han sido escasas.

\section{BIBLIOGRAFÍA}

1. Ley Orgánica 6/2001, de 21 de diciembre, de Universidades (B.O.E. de 24-XII-2001), pp. 49400-49425.

2. Comparecencia de la Ministra de Educación, Cultura y Deporte. Comisión de Educación, Cultura y Deporte. Cortes Generales Diario de Sesiones del Congreso de Los Diputados, Año 2001, VII Legislatura, Núm. 241 (29-V-2001), pp. 36203649 .

3. Comparecencia de la Ministra de Educación, Cultura y Deporte en la Comisión del Senado para informar sobre los borradores de los proyectos de Ley de Universidades y Formación Profesional (12-06-01). http://www.crue.org/leyuniversid.htm

4. Sesión Plenaria. Cortes Generales. Diario de Sesiones del Congreso de loa Diputados, Plenario y Diputación Permanente, Año 2001, VII Legislatura, Núm. 108 (27-IX-2001), pp. 52555280 .

5. Sesión del Pleno. Cortes Generales. Diario de Sesiones del Senado, Año 2001, VII Legislatura, Núm. 70 (13-XII-2001), pp. 4038-4131.

6. Intervención de la Ministra de Educación, Cultura y Deporte ante el Pleno del Consejo de Universidades (19-04-01). http: //www.crue-org/leyuniversid.htm.

7. Intervención de la Sra. Ministra sobre el Anteproyecto de Ley de Universidades en el Pleno del Consejo de Universidades (12-06-01). http://www.crue-org/leyuniversid.htm.

8. Informe sobre los aspectos de la Ley Orgánica de
Universidades cuya constitucionalidad es cuestionable (0201). http://www.crue-og/pdfr/DICT-CRUE-LOU06032002.pdf.

9. "El Consejo Consultivo de la Generalitat cree que la LOU es inconstitucional” El País, de 6-III-2002.

10. Pérez de Pablos S. El PSOE, IU y el Grupo Mixto recurre la LOU ante el Constitucional. El País, de 22-III-2002.

11. Datos generales del expediente 621/000037. http://www.senado.es/legis7/expedientes/expedientes/621000037.html.

12. Playà J. La Generalitat no recurrirá la LOU pese al informe de inconstitucionalidad del Consultiu. La Vanguardia, de 9-II2002.

13. Rusiñol P. CiU ignora el dictamen del Consultivo y descarta recurrir contra la LOU. El País, de 9-II-2002.

14. Europa Press. CiU impide llevar la LOU al Constitucional. La Vanguardia, de 28-II-02

15. Texto acordado por la Asamblea General de la CRUE (21-0501). http://www.crue-org/leyuniversid.htm.

16. Acuerdo de la Asamblea General de la CRUE (15-06-01). http://www.crue-org/leyuniversid.htm.

17. Nota sobre el Proyecto de Ley de Universidades dirigida a los grupos parlamentarios por la CRUE (19-09-01).

http://www.crue-org/leyuniversid.htm.

18. Proyecto de Real Decreto por el que se regula el sistema de habilitación nacional para el acceso a cuerpos de funcionarios docentes universitarios (03-02). http://www.grue.org/desarrolloLOUdocs/proyectoRDhabilitacion.htm.

19. Proyecto de Real Decreto por el que se regula el sistema de habilitación nacional para el acceso a cuerpos de funcionarios docentes universitarios (13-05-02). http://gestion.universia.es/ biblio-juridica/legislacion/borradores/borrador6.htm.

20. Peces-Barba G. Con la LOU a cuestas. El País , de 16-IV-02.

21. "Sesión Constituyente del Consejo de Coordinación Universitaria”. Comunidad Escolar, Núm.702. 2002.

22. "El Consejo de Estado emite dictamen favorable sobre el proyecto de Real Decreto de habilitación del profesorado universitario". Crónica Universia (19-VII-02).

23. Real Decreto 774/2002, de 26 de julio, por el que se regula el sistema de habilitación nacional para el acceso a Cuerpos de Funcionarios Docentes Universitarios y el régimen de los concursos de acceso respectivos (B.O.E. de 7- VIII-2002), pp. 29254-29268.

24. Solà C. Inédito e inaudito: el decreto de habilitación del profesorado. El País, de 21-X-2002.

25. Resolución de 31 de octubre de 2002, de la Secretaria General del Consejo de Coordinación Universitaria, por la que se convocan pruebas de habilitación nacional (B.O.E. de 6-XI-2002), pp. 32988-32993.

26. "Más de 2.000 candidatos concurren a 210 plazas de funcionarios docentes universitarios convocadas". Comunidad Escolar, Núm. 712. 2002.

27. Resolución de 13 de marzo de 2003, de la Presidencia del Consejo de Coordinación Universitaria, por la que se convocan 
pruebas de habilitación nacional (B.O.E. de 16-04- 03), pp. 15140-15144.

28. Resolución de 3 de julio de 2003, de la Secretaria General del Consejo de Coordinación Universitaria, por la que se convocan pruebas de habilitación nacional (B.O.E. de 30-VII-02), pp. 29570-29573.

29. Resolución de 31 de octubre de 2003, de la Secretaria General del Consejo de Coordinación Universitaria, por la que se convocan pruebas de habilitación nacional (B.O.E. de 25-XI2003), pp.41675-41680.

30. CRUE. Informe sobre las disfunciones detectadas en el sistema de habilitación nacional y su posible mejora (30-10-03). http://www.crue.org/pdf/asamcom habilitacion.pdf .

31. Tejada J. La habilitación a catedrático: luces y sombras. El País, de 12-I-04.

32. Ponsà M. Habilitaciones . El País , de 26-I-04.33. Martí J M. Entrevista a LLuís Ferrer Caubert, Rector de la Universidad Autónoma de Barcelona. El País, de 27-I-03.

33. Martí J. M. Entrevista Lluis Ferrer Caubet, Rector de la Universidad Autónoma de Barcelona. El País, de 27-I-03.

34. Pérez de Pablos S. Entrevista a Carlos Berzosa, Rector electo de la Universidad Complutense de Madrid. El País, de 31III-03.

35. Martí J M. Entrevista a Carles Solà, Consejero de Universidades de la Generalitat de Cataluña . El País, de 8-III-04.

36. Tusell J. Universidad: Ley mala, resultado previsible . El País, de 23-VI-03.

37. Sánchez C. La habilitación mueve a unos 90 profesores universitarios. El Adelanto de Salamanca, de 10-XII-03.

38. Martínez J P. Alternativa al sistema de habilitación. El País, de 18 -I-04.

39. Castillo J J. La inquisición sobre la habilitación. El País, de $10-\mathrm{V}-04$

40. FE,CC.OO. La habilitación del Profesorado. Universidad T.E. Hoja del Lunes , Núm. 273. 2003.

41. Pérez de Pablos S. La habilitación provoca que 1.500 docentes dejen sus clases durante un mes. El País, de 8-XII-03.

42. Pérez de Pablos S. Quejas en Sociología y Fisiología. El País, de 8-XII-03.

43. Buela-Casal G. El sistema de habilitación: proceso y criterios de evaluación. En "Curso Evaluación y acreditación de la calidad de la Educación Superior”. Almuñecar (09/04). http:// www.aneca.es/actividades/docs/eventos/almuo4_gualberto.ppt

44. Federación Nacional de Asociaciones de Catedráticos de Universidad. La insoportable levedad del ser la la L.O.U. Hoja Informativa (10-VI-02).

45. Tortella G. Todo queda en casa. El País, de 9-IV-01.

46. De Carreras F. Dos únics encerts per a la Universitat. El Periódico, de 16-VII-01.

47. Gaceta Universitaria. Especial LOU (28-XI-01).

48. Rivera M. La promoción del profesorado. La Razón , de 30-XI01.
49. Aguirregomezcorta M. Cara y cruz de la polémica ley de universidades. El País, de 17-XI-01.

50. Sesión del Pleno. Cortes Generales. Diario de Sesiones del Senado, Año 2001, Núm. 71 (14-XII-2001), pp. 4132-4181.

51. Michavila F. Mucho ruido y alguna nuez rancia. Gaceta Universitaria (7-V-01), p. 5.

52. Poves A. El azar y la Universidad. El País , de 25-V-01.

53. Contreras J. La L.O.U ¿son las alforjas que necesita la universidad para su viaje? Documento interno. Universidad de Barcelona. 2001.

54. Becerra J. La habilitación reabre el debate sobre la LOU. El Mundo Campus, de 18-XI-03.

55. Asenjo, M. Los rectores alertan sobre la "ineficiencia" del sistema de selección docente y apoyan su cambio. ABC, de 11-XI-03.

56. Europa Press .Educación y los rectores avaluarán la habilitación del profesorado para introducir posibles mejoras. El Mundo Campus, de 11-XII-2003.

57. "Una Comisión evaluará los resultados de la habilitación". ABC, de 16-XII-2003.

58. Becerra, J. Solo el $30 \%$ de las medidas para adaptarse a Europa afecta a la docencia. El Mundo Campus de 2-III-04.

59. Pérez de Pablos S. Zapatero se compromete ante los rectores a cambiar la ley de universidades y la reválida. El País, de 16-I-04.

60. Becerra, J. Las urnas dirimirán si sigue la LOU o se reforma. El Mundo Campus, de 9-III-04.

61. "Los rectores ofrecen su apoyo al próximo Gobierno para reformar la Ley Orgánica de Universidades. El Mundo Campus, de 15-IV-2004.

62. Proyecto de Ley Orgánica por la que se modifica la Ley orgánica 6/2001, de 21 de diciembre, de Universidades. Boletín Oficial de las Cortes Generales. Congreso de los Diputados, VIII Legislatura, Serie A, Núm. 101-1 (8-IX-06), pp. 1-16.

63. Documento de la Asamblea General de la CRUE sobre cambios en la LOU y orientaciones de las políticas universitarias (24-06-04) http://www.crue.org/leyuniversid.htm.

64. FE.CC.OO. CC.OO. demanda la modificación urgente del sistema de habilitación del profesorado universitario (30-06-04). http://www.uv.es/ssccoo/pdi/habilitacio.pdf.

65. AACTE. Reforma de la LOU: En defensa de la igualdad de oportunidades en la contratación del profesorado universitario (10/04). http://www. cica.es/aliens/aacte/documentos.html.

66. Intervención de la Ministra de Educación y Ciencia, $\mathrm{M}^{\mathrm{a}}$ Jesús San Segundo, en el pleno del Consejo de Coordinación Universitaria (28-07-04) http://www.mec.es/mecd/gabipren/ intervenc/edu_040728_coorduniv.pdf.

67. Ministerio de Educación y Ciencia. El Consejo de Coordinación Universitaria debate los decretos de Grado y postgrado. Nota de Prensa (20-10-04). http://www.mec.es/ mecd/gabipren/notas/2004/octubre/edu_25_Consejo.pdf

68. Crónica Universia .Hacia la convergencia Europea (27-10-04). http://www.universia.es/portada/actualidad/noticia_actualidad.jsp?noticia $=76100$ 
69. Real Decreto $338 / 2005$, de 1 de abril, por el que se modifica el Real Decreto 774/2002, de 26 de julio, por el que se regula el sistema de habilitación nacional para el acceso a cuerpos de funcionarios docentes universitarios y el régimen de los concursos de acceso respectivos (B.O.E. de 11-IV-2005), pp. 1241212414.

70. Resolución de 7 de septiembre de 2005, de la Secretaria General del Consejo de Coordinación Universitaria, por la que se convocan pruebas de habilitación nacional (B.O.E. de 21IX-2005), pp. 31386-31393.

71. CRUE. Nueva convocatoria de habilitación (14-09-05). http:// www.crue.org/pdf/nueva_convocatoria_habilitacion2.pdf

72. Resolución de 5 de marzo de 2004, de la Secretaria General del Consejo de Coordinación Universitaria, por la que se convocan pruebas de habilitación nacional (B.O.E. de 31-III2004), pp. 13707-13711.

73. Resolución de 5 de julio de 2004, de la Secretaria General del Consejo de Coordinación Universitaria, por la que se convocan pruebas de habilitación nacional (B.O.E. de 30-VII-2004), pp. 27622-27666.

74. Resolución de 2 de noviembre de 2004, de la Secretaria General del Consejo de Coordinación Universitaria, por la que se convocan pruebas de habilitación nacional (B.O.E. de 30XI-2004), pp. 39699-39703.

75. "El Consejo de Coordinación habilita 509 candidatos a distintos cuerpos docentes". El Mundo Campus, de 22-II-05.

76. Morán C, Benitez J. Educación eliminará con un decreto la prueba nacional para profesores universitarios. El País, de 26$\mathrm{X}-04$.

77. García $\mathbf{M}^{\mathrm{a}} \mathrm{A}$. Hay que diseñar un nuevo procedimiento de acceso a la función docente pública. En Encuentro de la CRUE en la UIMP "Las universidades en la sociedad del conocimiento" . CRUE Noticias, Núm 18 (2-09-04).

78. Pérez de Pablos S. Cada habilitado costará de media unos 9.000 euros. El País de 8-XII-03.

79. Estatuts de la Universitat Autònoma de Barcelona. http://www.crue.org/desarrolloLOUdocs/pdf/universidades/est atutos-superadoControl/_barcelona.pdf.

80. Resolución de 13 de mayo de 2004, de la Universidad Autónoma de Barcelona (B.O.E. de 4-VI-2004), pp. 2055420559.

81. Resolución de 3 de noviembre de 2004, de la Universidad Autónoma de Barcelona (B.O.E. de 30-XI-2004), pp.3970339708.

82. Estatut de la Universitat de Barcelona. http://www.crue.org/ desarrolloLOUdocs/pdf/universidades/estatutos-superado Control/ barcelonaweb.pdf.

83. Resolución de 6 de octubre de 2005, de la Universidad de Barcelona (B.O.E. de 26-X-2005 ), pp. 35100-35107.

84. Resolución de 6 de octubre de 2005, de la Universidad de Barcelona (B.O.E. de 3-XI-2005), pp. 36040-36043.

85. Estatuts de la Universitat de Girona. http://www.crue.org/
desarrolloLOUdocs/pdf/universidades/estatutos-superado Control/gironaweb.pdf.

86. Estatuts de la Universitat de Lleida. http://www.crue.org/ desarrolloLOUdocs/pdf/universidades/estatutos-superado Control/lleida.pdf.

87. Resolución de 17 de noviembre de 2005 de la Universidad de Lleida (B.O.E. de 2-XII-2005), pp.39789-39801.

88. Estatuts de la Universitat Politècnica de Catalunya. http:// www.crue.org/desarrolloLOUdocs/pdf/universidades/estatutos-superadoControl/politeccatalunyaweb.pdf.

89. Universitat Politècnica de Catalunya. Reglamento para los concursos de acceso a los cuerpos docentes universitarios. http://upc.es.

90. Estatutos de la Universitat Pompeu Fabra. http://www.crue. org/desarrolloLOUdocs/pdf/universidades/estatutos-superado Control/Pompeu.pdf.

91. Resolución de 3 de febrero de 2004, de la Universidad Pompeu Fabra (B.O.E. de 27-II-2004), pp. 9284-9287.

92. Estatut de la Universitat Rovira i Virgili. http://www. crue.org/desarrolloLOUdocs/pdf/universidades/estatutossuperadoControl/ roviraivirgili.pdf.

93. Resolución de 9 de octubre de 2006, de la Universidad Rovira Virgili (B.O.E. de 26-X-2006), pp.37403-37408.

94. Real Decreto 1052/2002, de 11 de octubre, por el que se regula el procedimiento para la obtención de la evaluación por la Agencia Nacional de Evaluación de la Calidad y Acreditación, y de su certificación, a los efectos de contratación de personal docente e investigador universitario. (B.O.E. de 12-X-2002), pp. 36095, 36096.

95. Resolución de 17 de octubre de 2002, de la Dirección General de Universidades, por la que se publican los criterios generales de evaluación de la Agencia Nacional de Evaluación de la Calidad y Acreditación para la contratación de personal docente e Investigador y se determina el procedimiento de solicitudes de evaluación o informe (B.O.E. de 30-X-2002), pp. 38222-38227.

96. "El personal docente e investigador puede presentar ya su solicitud para ser acreditado por la ANECA". Universia (8/11/02).http://www.universia.es.

97. ANECA. Programas/Evaluación del profesorado (04/03). http://www.aneca.es/modal_eval/profes_1.html.

98. ANECA. Programa de Evaluación del Profesorado. Memoria Febrero 2003-Enero 2004. (2004). http://www.aneca.es.

99. ANECA. Manual de Procedimiento para la emisión de evaluación o informe para la contratación de profesorado universitario (2005). http://www.aneca.es.

100. Asenjo M. La evaluación del profesorado contratado, nuevo foco de tensión en la Universidad. ABC, de 4-IV-2203.

101. EFE. CCOO denuncia casos de "arbitrariedad" en la evaluación de profesores. El Mundo Campus, de 22-V-2003.

102. EFE. Ismael Crespo dice que "no existe opacidad" en la evaluación del profesorado. El Mundo Campus, de 23-V-2003. 
103. Asenjo M. Los profesores contratados denuncian falta de transparencia y disparidad de criterios en la Agencia de Evaluación. ABC, de 21-X-2003.

104. FACU. Análisis crítico de la teoría y de la praxis de la "ANECA". Hoja Informativa (5-XI-2003).

105. M C. PSOE e IU piden el cese del director de la agencia que evalúa las universidades. El País, de 15-XI-2003.

106. Europa Press. Educación no destituirá al director de la ANECA, como pide la oposición. El Mundo Campus, de 19-XI-2003.

107. Agencias. Juan Vázquez recuerda la necesidad de mejorar la financiación universitaria. El Mundo Campus, de 25-XI-2003.

108. Castro D. La ANECA y los intereses creados. ABC, de 25-XI2003.

109. Agencias, Comisiones Obreras convoca movilizaciones contra el proceso de evaluación del profesorado. El Mundo Campus, de 26-XI-2003.

110. M A. Más de 1.500 docentes acusan a la ANECA de "oscurantismo". El País, de 28-XI-2003.

111. "Manifiesto: Un cambio necesario en la Universidad" (9/12/03). http://www.universia.es/contenidos/pdi/selección/ habilitacion.

112. "Profesores de medio centenar de Universidades apoyan una propuesta para cambiar la ANECA. ABC, de 20-I-2004.

113. "El Defensor del Pueblo cuestiona la "transparencia y objetividad" de la ANECA". El País, de 2-II-2004.

114. C M. El PSOE se queja ante el Defensor del Pueblo de la agencia que evalúa las universidades. El País, de 12-II-2004.

115. Resolución de 18 de febrero de 2005, de la Dirección General de universidades por la que se modifican determinados aspectos del procedimiento de presentación de solicitudes de evaluación o informe de la Agencia Nacional de Evaluación de la Calidad y Acreditación para la contratación de personal docente e investigador, así como los criterios de evaluación, establecidos en las Resoluciones de 17 de octubre de 2002 y de 24 de junio de 2003, de la Dirección General de Universidades. (B.O.E. de 4-III-2005), pp. 7875-7877.

116. ANECA. Informe del Programa de Evaluación del Profesorado para la contratación 2002-05. http://www.aneca.es.
117. "Agencias Autonómicas de Evaluación de la calidad". http://.www.universia.es/contenidos/pdi/seleccion/habilitacion/agencias_calidad.htm.

118. Llei 1/2003, de 19 de febrer, d'universitats de Catalunya (D.O.G.C. de 20-II-2003) pp-3326-3345.

119. AQU Catalunya. Evaluación del profesorado universitario. El profesorado agregado y catedràtico (2005). http://www.aqucatalunya.org.

120. AQUCatalunya. Informe sobre el desenvolupament del procés d'emissió de les acreditacions de recerca i de recerca avançada (2004) http://www.aqucatalunya.org.

121. AQU Catalunya. Evaluación del profesorado universitario. Certificación de la actividad docente (2005). http://www.aqucatalunya.org.

122. AQU Catalunya. Memoria de activitats 2004 (2005) http://www.aqucatalunya.org.

123. AQU Catalunya . AQU Catalunya certifica l'activitat docent del profesorat universitari (17-03-06). http://www.aqucatalunya.org.

124. Resolució de 10 d'ocyubre de 2006, de la Universitat Autònoma de Barcelona (D.O.G.C.de 17-XI-06), pp. 4821-4823.

125. Resolució de 17 de novembre de 2004, de la Universitat de Barcelona (D.O.G.C. de 3-XII-2004), pp. 24644-24647.

126. Resolució de 20 de maig de 2005, de la Universitat de Lleida (D.O.G.C. de 27-V-2005), pp. 15477-15482.

127. Universitat Politècnica de Catalunya. Reglament per a la provisió i contractació de Professorat Contractat Doctor. http:// www.upc.es.

128. Universitat Pompeu Fabra. Bases per concurs per a la provisió de places de personal acadèmic. Professor catedràtic contractat. http://www.upf.edu/rrhh/acces/pdi/catedratic/bases.htm.

129. Universitat Pompeu Fabra. Bases per concurs per a la provisió de places de personal acadèmic. Professor agregat. http:// www.upf.edu/rrhh/acces/pdi/agregats/bases.htm.

130. Universitat Rovira I Virgili. Normativade concursos per a la contractació de professorat. http://www.urv.es/la_urv/10_serveis/rrhh/PDI/Normatives. 